\title{
A Novel Design of Needle Aspiration Biopsy Monitoring Instrument (NAOMI) Tested on a Low Cost Chest Phantom
}

\author{
Rino Ferdian Surakusumah ${ }^{1}$, Muhammad Qurhanul Rizqie ${ }^{1}$, Dwichayo Herlambang Prabowo Hidayat ${ }^{3}$, Eko Supriyanto ${ }^{2}$, \\ and Muhammad Haikal Satria ${ }^{2}$ \\ ${ }^{1}$ Faculty of Biosciences and Medical Engineering, Universiti Teknologi Malaysia, Johor Bahru, 81310 Malaysia \\ ${ }^{2}$ IJN-UTM Cardiovascular Engineering Center, Institute of Human Centered Engineering, Universiti Teknologi Malaysia, Johor Bahru, 81310 \\ Malaysia \\ ${ }^{3}$ Institut Teknologi Bandung, Bandung, West Java, 40132 Indonesia
}

\begin{abstract}
Needle biopsy is a medical intervention method for taking a lung tissue sample that suspected as a cancer. The disadvantage is the physicians directly visualize the anatomical structures in an open surgery for lung cancer biopsy procedure. There is a need to develop an instrument that may help the physician to guarantee the accuracy and efficiency while performing needle aspiration biopsy. Therefore, a needle aspiration biopsy monitoring instrument or named as NAOMI is proposed. It consists of a microcontroller system, an IMU sensor, an ultrasonic ranging module, a bluetooth module, and a $9 \mathrm{~V}$ lithium battery. The experimental testing consist of performance testing, functional testing using chest phantom, and user acceptances. The results showed that the NAOMI improve the accuracy and efficiency while performing the needle biopsy operation.
\end{abstract}

\section{Intoruction}

Needle aspiration biopsy is a one of medical intervention method for taking a lung tissue sample that suspected as a cancer from a medical imaging procedure. It uses a long needle or known as biopsy needle inserted through the chest wall to the target of lung tissue sample. This method is used if the abnormal lung tissue is located close to the chest wall. A computed tomography (CT) scan, an ultrasound, or fluoroscopies are usually used to guide the needle to the abnormal tissue [1].

The disadvantage of these mentioned methods is the physicians directly visualize the anatomical structures in an open surgery for lung cancer biopsy procedure. The physician mentally recreates the spatial relationship between the surgical tools and anatomy based on the single or multiple 2D images. The outcome is highly dependent upon the physicians skills and experiences to mentally reconstruct a 3D scene, which in turn leading to inter and intra-observer variability. Accuracy and efficiency are predominant doubt in these cases. Besides, existing Computed Tomography (CT) Fluoroscopy also exposes both physician and patients to high doses of radiation, the longer time the procedure the greater damage may incurred [2], [3].

From this background, there is a need to develop an instrument that may help the physician to guarantee the accuracy and efficiency while performing needle aspiration biopsy. Therefore, a needle aspiration biopsy monitoring instrument or named as NAOMI is proposed. It is integrated with an image guided navigation system and aims to obtain orientation and depth information of the biopsy needle. It may shorten the learning period required for minimally invasive procedures and reduce the variability of the outcome, narrowing the gap between exceptional and standard practice without exposing to any doses of radiations. They may enable new minimally invasive procedures allowing physicians to perform procedures that were previously considered too dangerous.

\section{Material and Method}

\subsection{Concept}

In the last few years, there are some research that were conducted to enhance the accuracy of lung needle biopsy operation. In 2013, Zhou et al. [4], developed a robotic biopsy system that has capability to adapted to the patient breathing motion pattern. It obtained several images from the patient lung in breathing cycle then combined with a camera that recorded the patient skin surface to recognized breathing phase. In 2014, Surakusumah, RF et al. [5], [6], developed a novel design of flexible bronchoscopy for lung bronchoscopic biopsy using soft actuator mechanism. In 2015, Yaffe, D et al. [7], developed a novel technique for CT-guided transthoracic biopsy of lung lesions. It used a coaxial CT-guided TNAB while a 22-gauge guide wire was used to locate the lesion of the biopsy accurately. Based on authors knowledge, there was no study that improved the 
accuracy using orientation combined with depth measurement approach. Therefore, a Needle Aspiration Biopsy Monitoring Instriment or named as NAOMI that can guide a physician to know orientation and depth of a biopsy needle is proposed.

Based on study from Fadzil et al. [8] on 2015, orientation can be measured using Inertial Measurement Unit (IMU) sensor. It is relatively small and does not require sensor setup in different distances compared to other accelerometer system. Based on Dey, R et al. [9] who studied about expansion measurement of digital chest, an ultrasonic sensor was used to detected distance from any object to the surface of human body. Thus, an IMU and an ultrasonic sensors was intergrated to the NAOMI.

From the information in Haydock, MD et al. [10], there is a requirement for medical instruments to have evaluation of user acceptances. It is related to the ergonomic design concept of the medical instruments. So, the NAOMI design followed the ergonomic concept and be evaluated qualitatively by the possible users such as ordinary person, radiologist, and medical doctors. To measured the performance of the NAOMI, a lung nodule phantom that developed by Yusof NSM et al. [11] was used.

\subsection{Design and fabrication}

Design of the NAOMI consists of embedded system, and casing. The embedded system integrate a microcontroller system (ARDUINO Mini Pro), an IMU sensor (The InvenSense MPU-6050), an ultrasonic ranging module (HC-SR04), a bluetooth module (HC-06), and a 9V lithium battery. The IMU sensor obtained pitch and yaw position datas of the NAOMI with 10 resolution. The ultrasonic ranging module obtained the distance between the NAOMI front side (base point of the biopsy needle) and patient chest surface with $3 \mathrm{~mm}$ resolution. A bluetooth module bridge the data transfer from the NAOMI to a 2D X-ray Lung Biopsy Software. In order to powered the NAOMI embedded system, the 9V lithium battery was connected to RAW pin of the microcontroller system that contain a feature to accepted an unregulated power supply. The NAOMI embedded system schematic is shown in Fig. 1. The fabrication of the PCB and component setup was conducted.

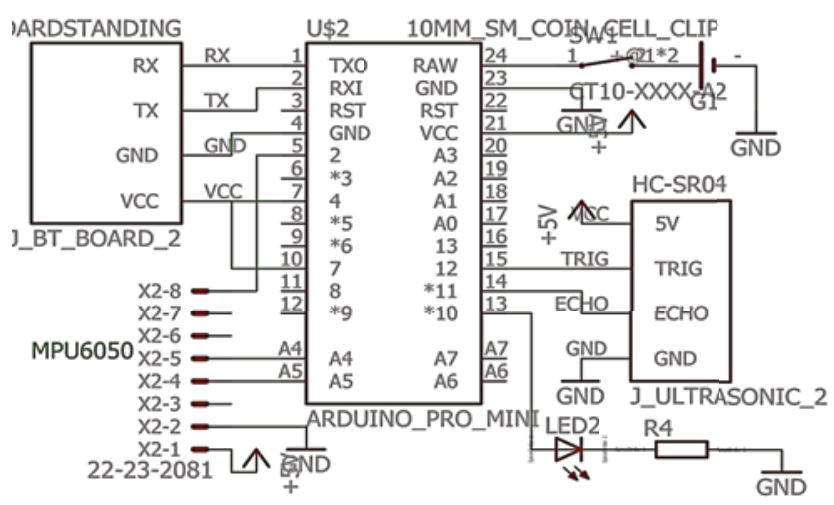

Figure 1. The NAOMI embedded system schematic.

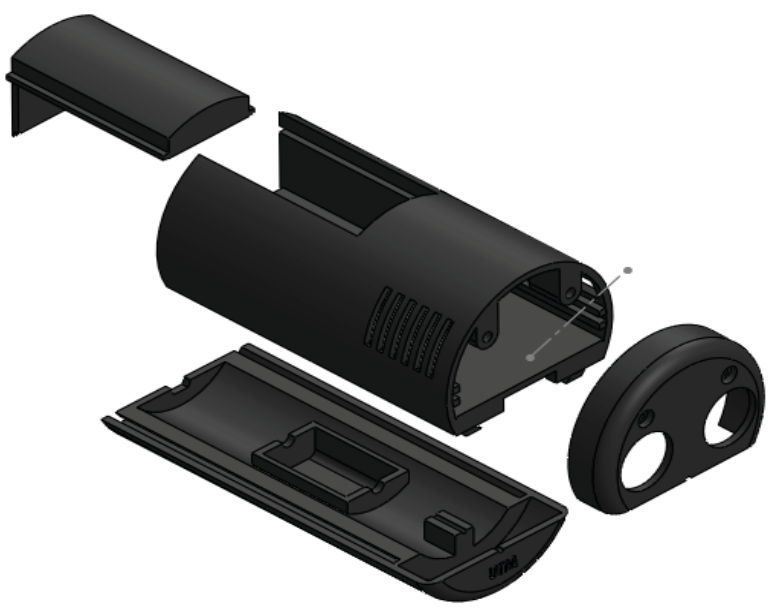

Figure 2. The NAOMI casing 3D design

The NAOMI casing was designed using rapid prototyping (Solidworks) software. It has cylindrical shaped with $50 \mathrm{~mm}$ diameter and $105 \mathrm{~mm}$ length size in order to provide an optimum handling for the user. A placement of biopsy needle was designed in such a way that the user is able to replace the biopsy needle. As a note, this design only suitable for NAC-1825B-BX type of biopsy needle. The NAOMI casing 3D design is shown in Fig. 2. It was fabricated using 3D printer machine (MakerBot) with Acrylonitrile Butadiene Styrene (ABS) material.

\subsection{Experiment testing}

The testing of the NAOMI consisted of performance, functional, and user acceptance. The performance consisted of orientation and depth measurement. Orientation measurement was conducted using a camera that visualized the rotation in row and pitch of the NAOMI in front of an angle measurement sheet. As comparations, the NAOMI was mounted to a motorized orientation measurement device that gave the orientation data from the output of motors and also the orientation data which transferred to the 2D X-Ray Lung Biopsy software. The depth was measured using the camera that visualized the distance between the NAOMI to the object in front of a milimeter ruler. The depth data that transferred to the 2D X-Ray Lung Biopsy software was used for the comparation. The NAOMI performance testing setup is shown in Fig. 3.

In conjuction with 2D X-Ray Lung Biopsy software and using a lung nodule phantom as an object experiment, a functional system was tested. After an optimum orientation and a depth data of targeted lung nodule was collected by the software, the NAOMI mounted with a biopsy needle was prepared by the user. The tip of the biopsy needle was positioned at a hole of a red tape that indicated the starting point of needle insertion. The NAOMI transfered the biopsy needle orientation and depth information to the software. The user adjusted the NAOMI orientation in such a way that the actual NAOMI orientation is similar with the orientation that visualized in the software While holding the NAOMI, the user inserted the biopsy needle into the 


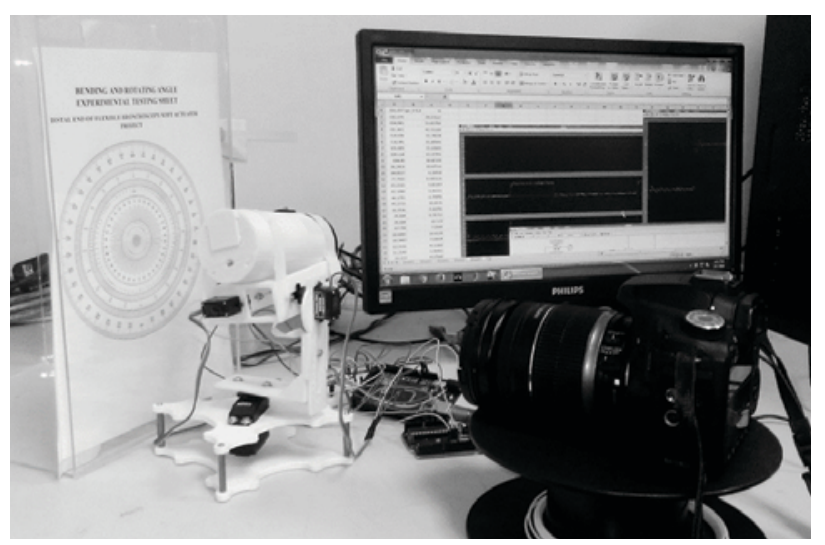

(a)

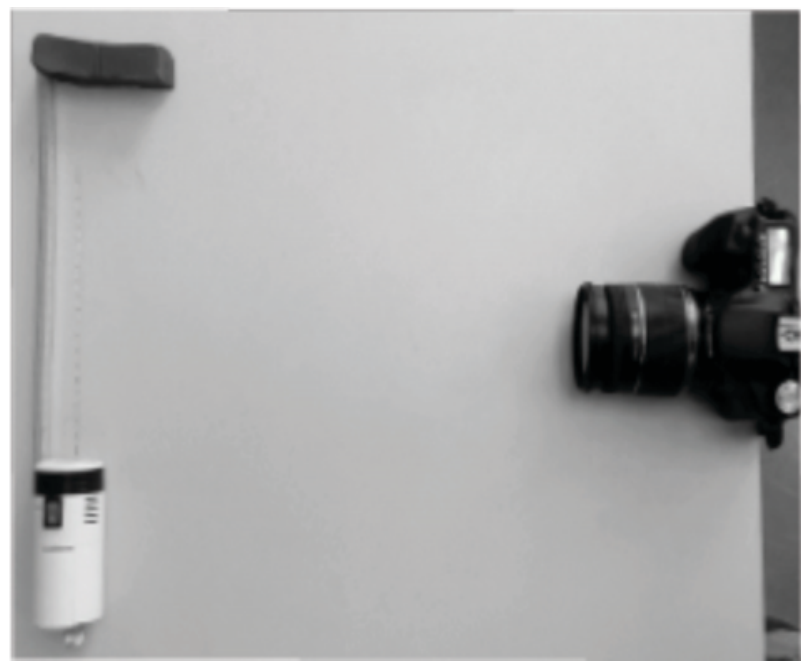

(b)

Figure 3. The NAOMI performance testing setup: (a) Orientation, (b) depth

lung nodule phantom until the depth reach the target that visualized in the software. After the biopsy needle reach the target, a sample taker needle was inserted through the biopsy needle in such a way that it reached the lung nodule and a sample will be taken out from the biopsy needle. Because of the lung nodule phantom provided different material of the lung nodule compared to the other tissue and by some numbers of experiment, the data of how accurate and fast the NAOMI guide the biopsy needle to take the tissue sample can be measured. The NAOMI functional testing setup is shown in Fig. 4.

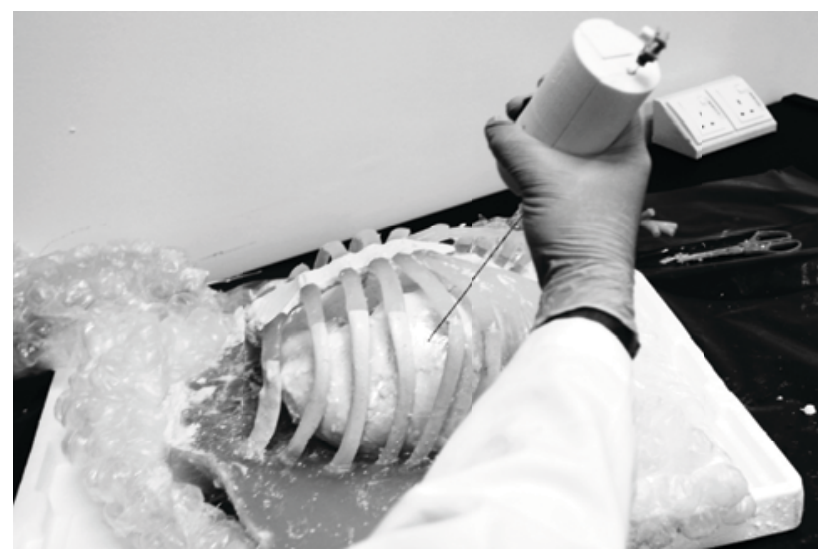

Figure 3. The NAOMI functional testing setup
For obtained the evaluation of user acceptances and ergonomic value of the NAOMI, a questionnaire was designed and distributed to 5 ordinary persons, 5 radiologists, and 5 medical doctors. The questionnaire consists of user personal information such as experiences in radiology and pulmonology, and procedural information such as evaluation in prototype demo, ergonomic design, and accuracy.

\section{Result and discussion}

Fig. 3 and Fig. 4 shows the final NAOMI prototype, which has a total length of $105 \mathrm{~mm}$ and a diameter of $50 \mathrm{~mm}$. The lower part consists of a biopsy needle placement box (BNPB) that fit to the NAC-1825B-BX main housing part. The BNPB positioned about $32 \mathrm{~mm}$ from the back surface of NAOMI in order to set the difference between biopsy needle tip and sample-taker needle position of $3 \mathrm{~mm}$. This is designed so that tissue sample cutting may be operated effectively. To combine the lower part and the upper part, a specific-shape connector is designed in both connecting surface of the part. This prototype configuration offered the optimum in user-friendliness and needle biopsy operation.

\subsection{Performance result}

The graph of orientation measurement experimental result is shown in Fig. 5. The graph consists of testing number of different angle position of pitch and yaw and different measurement methods which are from camera, motorized orientation sensor, and NAOMI depth sensor. The pitch measurement refer to $00-350$ o line while the yaw measurement refer to $3500-00$. Using mean square error (MSE) analysis, the test show an optimum accuracy with 0.063 error.

The graph of depth measurement experimental result is shown in Fig. 6. The graph consists of testing number of different distance from front side of the NAOMI until the chest surface in milimeters. Using mean square error (MSE) analysis, the test show an optimum accuracy with 10 error.

\subsection{Functional result}

From 10 trials of the lung needle biopsy operation using NAOMI in conjuction with the 2D X-ray lung needle biopsy software, the result shows that there was not more than 1 minutes with $100 \%$ successful rate in obtaining the tissue sample at the first trial

\subsection{User acceptance}

Meetings with physicians provided important feedback concerning functional procedure and power handling issue of the NAOMI. The physicians was asked to operate an in-vitro lung needle biopsy procedure with the chest phantom without use NAOMI and use NAOMI in conjunction with 2D X-ray Lung Biopsy software. The NAOMI is improve the accuracy of the physician to 
conduct needle biopsy operation. It is lightweight and has a depression in the handle during operation. This was introduced after learning that the way physician conducted the lung neddle biopsy procedure without using NAOMI and with using NAOMI in conjunction with 2D X-ray Lung Biopsy software. The goal was to provide the NAOMI as ergonomic as well so the physicians would feel comfortable using the device.

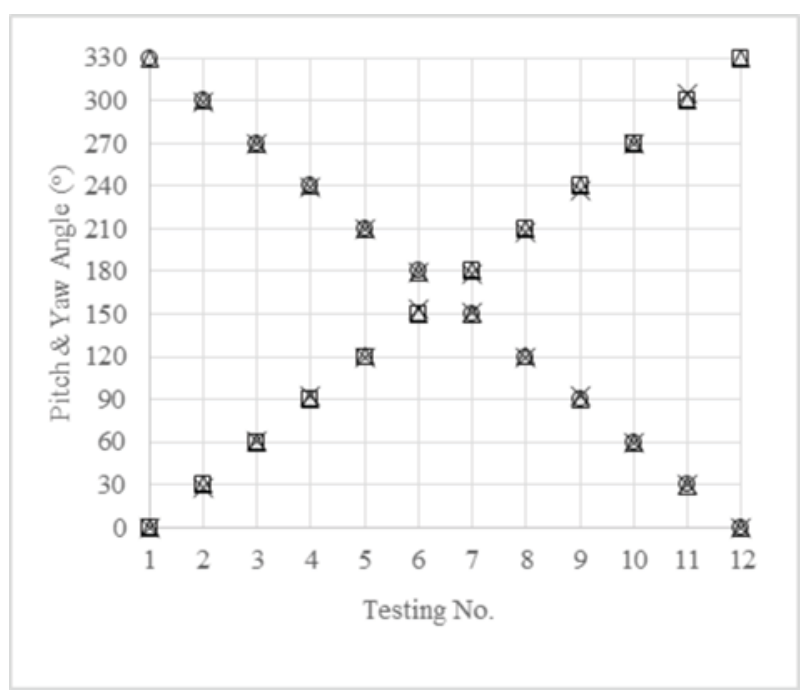

Figure 5. Orientation Measurement Experimental Result

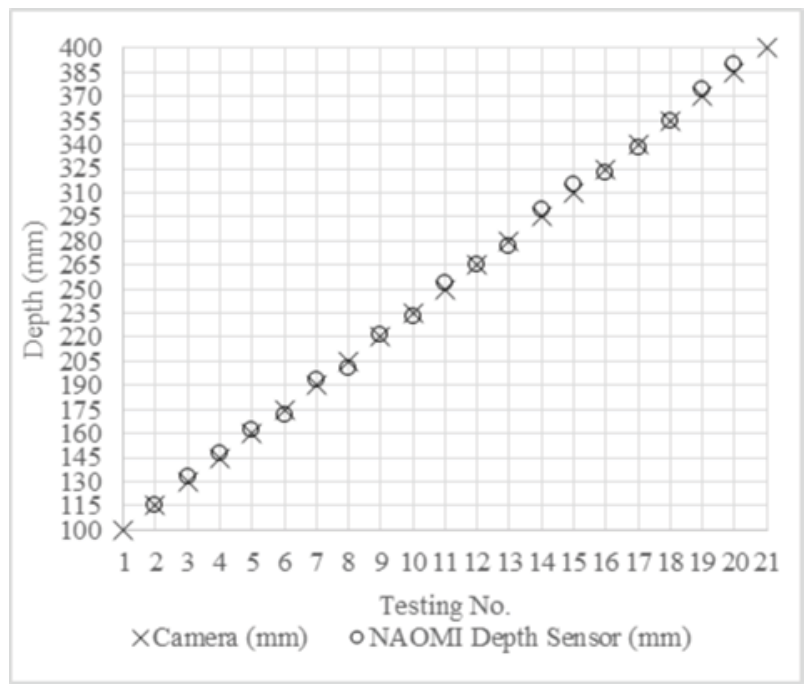

Figure 6. Depth Measurement Experimental Result

\subsection{Discussion}

Related to the performance of the NAOMI, based on the depth measurement result, the error was quite significant. It is expected because of the ultrasonic ranging module resolution and the orientation between the NAOMI and the chest surface. A higher resolution distance sensor may improve the accuracy. The orientation measurement already performed an optimum accuracy in determining the position of the NAOMI.
Related to the functional measurement and the user acceptances, the NAOMI in conjuction with 2D X-ray Lung Biopsy Software is able to guide the physiscian to orientate the biopsy needle in such a way that has optimum accuracy to take the tissue sample. It also able to measure the depth of the biopsy needle. However, during the operation, a skill and a human ability factor of the physician still importantly needed. When the physician is not well-trained enough for stabilizing the orientation of the NAOMI until it reach the target, the target will be possibly different. An equipment that able to fix the position of the biopsy needle or the use of robot may solve the issue. The lower part of the NAOMI may also redesign in such a way that it fit to other type of biopsy needle..

\section{Conclusion}

A novel design of Needle Aspiration Biopsy Monitoring Instrument (NAOMI) was developed for monitoring the lung needle biopsy by providing the orientation and depth information in in-vitro study. With considering the ergonomic design and using the NAOMI in conjuction with 2D X-Ray Lung Biopsy software, it is expected to help the physicians to improve accuracy and efficiency while performing the needle biopsy operation. In the future, further study on optimizing the design, accuracy, and the experiment using cadaver and also the clinical testing will be conducted to justify the development of the NAOMI.

\section{Acknowledgement}

This research was supported by the Ministry of Science, Technology and Innovation (MOSTI) Malaysia Grant No. 02-01-06-SF1191 (R.J130000.7909.4S091).

\section{References}

1. L. G. Collins, C. Haines, R. Perkel, and R. E. Enck, "Lung cancer: Diagnosis and management," Am. Fam. Physician, vol. 75, no. 1, pp. 56-63, 2007.

2. Y. Y. Cao, N. Fan, F. Xing, L. Y. Xu, Y. J. Qu, and M. Y. Liao, "Computed tomography-guided cutting needle pleural biopsy: Accuracy and complications," Exp. Ther. Med., vol. 9, no. 1, pp. 262-266, 2015.

3. C. M. Ogilvie, J. T. Torbert, J. L. Finstein, E. J. Fox, and R. D. Lackman, "Clinical utility of percutaneous biopsies of musculoskeletal tumors.," Clin. Orthop. Relat. Res., vol. 450, no. 450, pp. 95$100,2006$.

4. Y. Zhou, K. Thiruvalluvan, L. Krzeminski, W. H. Moore, Z. Xu, and Z. Liang, "CT-guided robotic needle biopsy of lung nodules with respiratory motion - Experimental system and preliminary test," Int. J. Med. Robot. Comput. Assist. Surg., vol. 9, no. 3, pp. 317-330, 2013. 
5. M. Automation, R. F. Surakusumah, D. Ekashanti, O. Dewi, E. Supriyanto, and M. Engineering, "Development of a Half Sphere Bending Soft Actuator for Flexible Bronchoscope Movement," pp. 120-125, 2014.

6. A. M. Faudzi, R. F. Surakusumah, D. E. O. Dewi, I. N. A. M. Nordin, and M. R. M. Razif, "Development of Flexible Bronchoscope Device Using Soft Actuator," in Medical Imaging Technology, Springer Singapore, 2015, pp. 223-241.

7. D. Yaffe, M. Koslow, H. Haskiya, and D. Shitrit, "A novel technique for CT-guided transthoracic biopsy of lung lesions: improved biopsy accuracy and safety,” Eur. Radiol., vol. 25, no. 11, pp. 3354-3360, 2015.

8. D. E. Octorina Dewi, M. Mohd. Fadzil, A. `Athif Mohd. Faudzi, E. Supriyanto, and K. W. Lai, "Position Tracking Systems for Ultrasound Imaging: A Survey," in Medical Imaging Technology Reviews and Computational Applications, W. K.
Lai and E. D. Octorina Dewi, Eds. Singapore: Springer Singapore, 2015, pp. 57-89.

9. R. Dey, U. Thakur, L. Sunny, L. D'Almeida, and K. Chakravarty, "E-Health and Bioengineering Conference (EHB), 2015," E-Health and Bioengineering Conference (EHB), 2015. pp. 1-4, 2015.

10. M. D. Haydock, A. Mittal, C. F. Wilkes, D. H. Lim, E. Broadbent, and J. A. Windsor, "Interaction Between Objective Performance Measures and Subjective User Perceptions in the Evaluation of Medical Devices: a Case Study," Int. J. Technol. Assess. Health Care, vol. 31, no. 05, pp. 297-303, 2015.

11. M. Q. Rizqie, N. S. M. Yusof, R. F. Surakusumah, D. E. O. Dewi, E. Supriyanto, and K. W. Lai, "Review on Image Guided Lung Biopsy," in Medical Imaging Technology, Springer Singapore, 2015, pp. 41-56. 